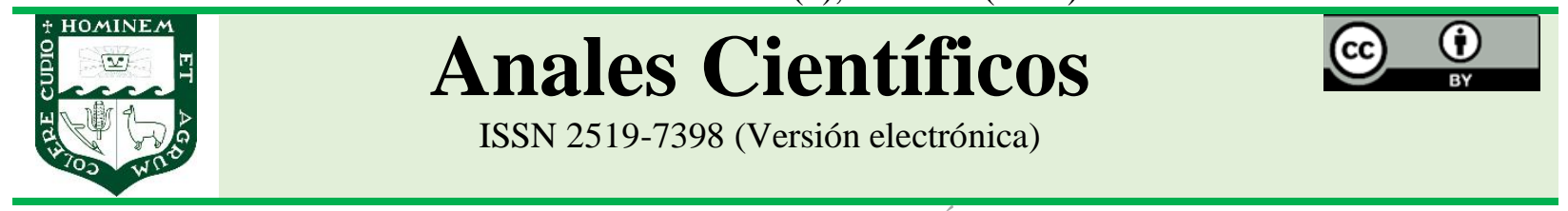

ARTÍCULO ORIGINAL - RESEARCH ARTICLE http://dx.doi.org/10.21704/ac.v82i1.1747

\title{
MAPEO DE SERVICIOS ECOSISTÉMICOS CULTURALES EN ARUBA DEL SUR DEL CARIBE
}

\section{Mapping cultural ecosystem services in Aruba the Southern Caribbean}

\author{
Vanessa Sofía Soberón-Forsberg $1 *$ (i) \\ ${ }^{1}$ Facultad de Ciencias, Universidad Nacional Agraria La Molina, La Molina, 15024, Lima, Perú. \\ * E-mail: vsoberon@lamolina.edu.pe
}

Recibido: 15/05/2020; Aceptado: 05/08/2021; Publicado: 31/08/2021

\begin{abstract}
The objective study was to map cultural ecosystem services (aesthetic appreciation and recreation) in Aruba, from tourists' point of view. The Public participation Geographical Information System was used. Aruba has two types of visitors: Stay-Over visitors (who spend at least one night in Aruba) and Cruise visitors (those who do not spend the night). Data was obtained by interviewing tourists using Public Participatory Geographic Information System in October and November 2016 and March 2017. The data was used to determine tourists' aesthetic and recreational hot spots (areas with an abundance of events) and then to compare Cruise visitors' hot spots with Stay-Over visitors' hot spots. Then, tourists' hot spots were compared with the local resident's hot spots. The residents' data was obtained from a previous study. Tourists and local citizens indicate that the California Lighthouse was an aesthetic hotspot and that Eagle Beach was a recreational hotspot. Tourists and local citizens disagree about Palm Beach and Arikok National Park aesthetic value and about Surfside Beach recreational value. It is concluded that Eagle Beach, Palm Beach and California Lighthouse are areas of great interest and that there are similarities and differences in stakeholder perception of Aruba's aesthetic and recreational hotspots.
\end{abstract}

Keywords: Cultural ecosystem services; public participatory mapping; tourists

RESUMEN

El objetivo del estudio fue determinar los lugares donde los turistas experimentan mayor satisfacción de los servicios ecosistémicos culturales (recreación y apreciación estética) en Aruba. Se utilizó la metodología Sistema de Información Geográfica Participativos para mapear los servicios ecosistémicos. Aruba tiene dos tipos de turistas: turistas de estancia (que pasan al menos una noche en Aruba) y turistas de cruceros (aquellos que no pasan la noche). Los datos se obtuvieron mediante entrevistas a los turistas utilizando el mapeo participativo en octubre y noviembre de 2016 y marzo de 2017. Se utilizaron datos de mapeo participativo de los ciudadanos locales de un estudio anterior. Se determinó los puntos críticos (zonas con abundancia de eventos) estéticos y recreativos de los turistas. Se comparó los puntos críticos de los turistas con los puntos críticos de los residentes locales y los puntos críticos de los turistas de crucero con los turistas de estancia. Los turistas y ciudadanos locales indicaron que California Lighthouse fue un punto crítico estético y que Eagle Beach fue un punto crítico recreativo. Los turistas y ciudadanos locales estuvieron en desacuerdo respecto al valor estético de Palm Beach y el Parque Nacional Arikok y respecto al valor recreativo de Surfside Beach. Se concluye que Eagle Beach, Palm Beach y California Lighthouse 
son zonas de mucho interés y que hay similitudes y diferencias en la percepción de las partes interesadas sobre los puntos críticos estéticos y recreativos de Aruba.

Palabras clave: Servicios ecosistémicos culturales; mapeo participativo; turistas

Forma de citar el artículo (Formato APA):

Soberón-Forsberg, V. (2021). Mapeo de servicios ecosistémicos culturales en Aruba del Sur del Caribe. Anales Científicos. 82(1), 106-121. http://dx.doi.org/10.21704/ac.v82i1.1747

Autor de correspondencia (*): Vanessa Soberón-Forsberg. Email: vsoberon@ lamolina.edu.pe

(C) Los autores. Publicado por la Universidad Nacional Agraria La Molina.

This is an open access article under the CC BY

\section{INTRODUCCIÓN}

Existe una conexión entre los ecosistemas y el bienestar humano, el cual está dado por los servicios ecosistémicos. Estos son la contribución de la naturaleza al bienestar humano (seguridad, materiales esenciales para una vida decorosa, la salud, las buenas relaciones sociales y la libertad de elección y acción), los cuales se pueden apreciar en la Figura 1 (TEEB, 2010a). Los servicios ecosistémicos se clasifican en cuatro categorías: los servicios de aprovisionamiento los cuales son la cantidad de bienes o materias primas que un ecosistema ofrece (por ejemplo, alimentos y agua dulce); los servicios de regulación los cuales son los servicios que realizan las funciones clave de los ecosistemas (por ejemplo, la regulación de la calidad del aire); los servicios culturales que son los beneficios no materiales que las personas obtienen de los ecosistemas (por ejemplo, los servicios recreativos) y los servicios de apoyo los cuales realizan los procesos ecológicos necesarios para los otros $3 \mathrm{SE}$ (por ejemplo, fotosíntesis) (Millennium Ecosystem Assessment, 2005) y (TEEB, 2010a).

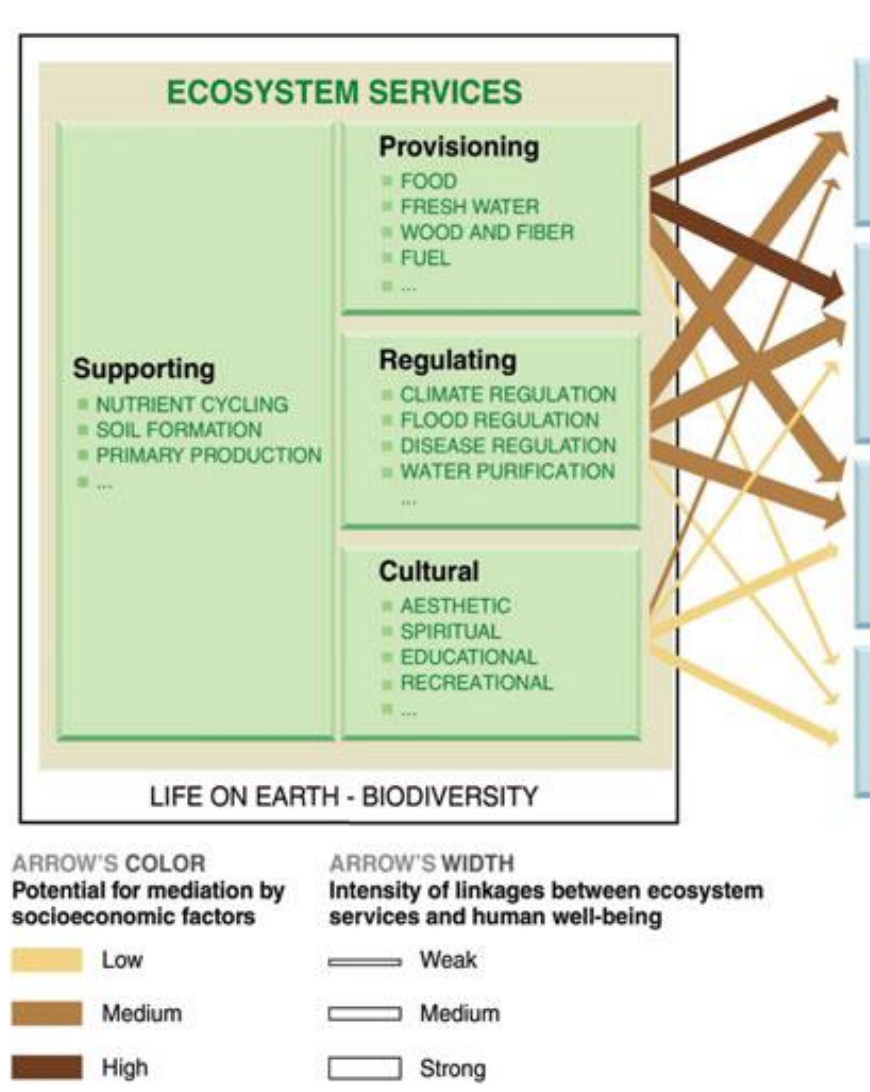

\section{CONSTITUENTS OF WELL-BEING}

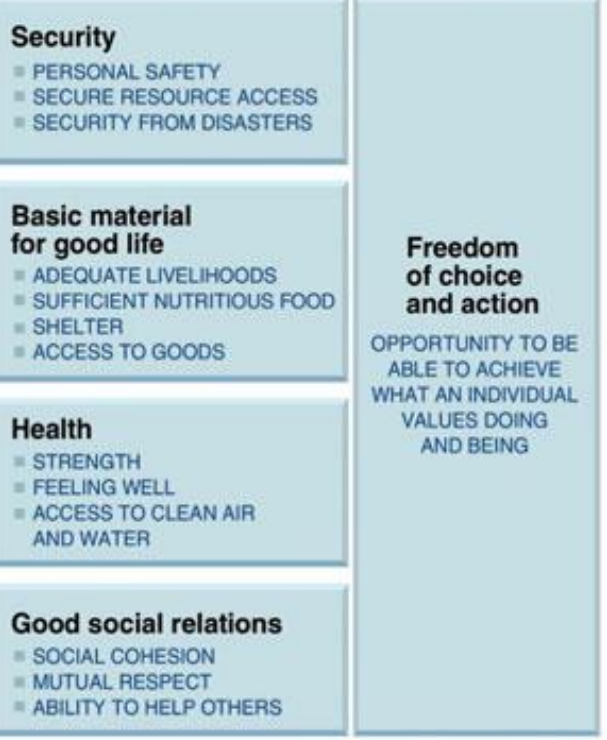

Source: Millennium Ecosystem Assessment

Figura 1. Vínculos entre los servicios ecosistémicos y el bienestar humano (Millennium Ecosystem Assessment, 2005). 
Es difícil de comprender la contribución de los servicios ecosistémicos culturales al bienestar humano porque la conexión entre los SE culturales y el bienestar humano es baja (Figura 1), ya que los SE culturales son intangibles y a menudo su valor no es reflejado en las transacciones del mercado. Una forma de estudiar la contribución de los servicios ecosistémicos culturales al bienestar humano es mediante el mapeo participativo. Este se define como una modalidad para registrar especialmente los valores sociales de los SE. Para comprender el valor de los servicios ecosistémicos es necesario integrar la perspectiva y las necesidades de las personas (Scholte et al., 2015) y esto puede realizarse utilizando los sistemas de información geográfica para mapear los servicios ecosistémicos.

El mapeo participativo se utiliza para identificar lugares donde se pueden encontrar o experimentar ciertos valores socioculturales (Scholte et al., 2015). En este método se le pide a los encuestados que señalen lugares donde se pueden encontrar ciertos valores colocando puntos en áreas como una forma de mapeo participativo (Scholte et al., 2015). En el mapeo participativo se les pregunta a los encuestados dónde disfrutan de los servicios culturales (Scholte et al., 2015). Estos métodos utilizan la tecnología de Sistemas de Información Geográfica (SIG) para identificar SE y reúnen el conocimiento y las experiencias de las partes interesadas sobre las características del medio ambiente utilizando un enfoque de abajo hacia arriba (Plantin, 2014). Los métodos del sistema de información geográfica de mapeo participativo van desde las aplicaciones en papel (los participantes identifican atributos y ubicaciones en un mapa físico utilizando marcadores o pegatinas) hasta las aplicaciones informáticas (los participantes identifican las ubicaciones utilizando herramientas digitales) (Brown et al., 2012).

Estudios anteriores descubrieron que las características de las partes interesadas pueden afectar a la percepción de los servicios ecosistémicos. Un estudio indica que las variables como la ubicación geográfica de los participantes, las clases sociodemográficas, las creencias, los valores y la ideología de los participantes y los conocimientos/experiencia en la zona de estudio puede afectar los resultados del mapeo participativo de los servicios ecosistémicos (Brown \& Kyttä, 2014). De igual forma, las características de los encuestados, como el género, la edad, el nivel de educación y el conocimiento del paisaje también pueden influir en los resultados del sistema de información geográfica del mapeo participativo (Brown \& Reed, 2009). Además, el origen de las partes interesadas afecta a las percepciones de los servicios ecosistémicos culturales (Plieninger et al., 2013). Por otro lado, hay estudios que indican que las partes interesadas tienen diferentes intereses y percepciones en el uso de los servicios ecosistémicos. Por ejemplo, un estudio analiza los servicios recreativos de los manglares de Sundarbans en Bangladesh e indica que los turistas y los usuarios locales perciben de forma diferente los entornos naturales (Chakraborty et al., 2020). Para los ciudadanos locales la recreación es una actividad más espontánea y no está asociada con gastar dinero mientras que para los turistas la recreación necesita la planificación y el uso de capital económico (Chakraborty et al., 2020). Otro estudio de servicios ecosistémicos de un humedal en Holanda analiza las escalas espaciales de los servicios de los ecosistemas (Hein et al., 2006). Se valoran cuatros servicios ecosistémicos brindados por los humedales: la provisión de caña para cortar, la provisión de peces, recreación y conservación de la naturaleza (Hein et al., 2006). La corta de juncos y la pesca solo son importantes a escala municipal, la recreación es más relevante a escala municipal y provincial y la conservación de la naturaleza es importante a nivel nacional e internacional (Hein et al., 2006). El estudio demuestra que en diferentes escalas las partes interesadas tienen intereses muy diferentes en los servicios de los ecosistemas (Hein et al., 2006).

Un estudio indica que los turistas, el personal del parque y los ciudadanos locales tienen un uso diferente de los servicios ecosistémicos culturales y de aprovisionamiento de un parque natural en el Brasil (Ribeiro \& Katia, 2016). Otro estudio indica que las partes interesadas de baja y alta influencia tienen percepciones diferentes de la distribución espacial de los servicios ecosistémicos culturales (García Nieto et al., 2015). Las partes interesadas de alta influencia se definieron como aquellos que tienen un alto grado de interés en el estado de los 
servicios de los ecosistemas y una influencia importante en el proceso de toma de decisiones ambientales (García Nieto et al., 2015), mientras que las partes interesadas de baja influencia se definieron como aquellos que tienen un alto grado de interés en el estado de los servicios de los ecosistemas y una baja influencia en la gestión ambiental (García Nieto et al., 2015).

El objetivo general del estudio fue determinar los lugares donde los turistas experimentan mayor satisfacción de los servicios ecosistémicos culturales (recreación y apreciación estética) en Aruba, utilizando los Sistema de Información Geográfica Participativo (SIGP). Para lograr este objetivo se buscó: (i) identificar los lugares donde los turistas experimentan los valores más altos de recreación utilizando los Sistema de Información Geográfica Participativo (SIGP), (ii) identificar los lugares donde los turistas experimentan los valores más altos de apreciación estética utilizando los Sistema de Información Geográfica Participativo (SIGP). Adicionalmente, se consideró importante determinar si hay una diferencia en los lugares donde se experimenta mayor satisfacción de los servicios ecosistémicos culturales (recreación y apreciación estética) de acuerdo con la percepción de las diferentes partes interesadas (turistas o ciudadanos locales) en Aruba. Este objetivo consistió en (iii) comparar los datos obtenidos de los turistas con los datos del Sistema de Información Geográfica Participativo obtenidos mediante una encuesta a los ciudadanos locales en un estudio anterior (Bubalo, 2016); para determinar si existen diferencias entre el patrón de lugares con valores más altos de recreación y apreciación estética de los ciudadanos locales, por un lado, y turistas, por otro.

\section{MATERIALES Y MÉTODOS}

Para cumplir con el objetivo de determinar los lugares donde los turistas experimentan mayor satisfacción de los servicios ecosistémicos culturales (recreación y apreciación estética) se seleccionó el método de mapeo participativo porque el mapeo los servicios ecosistémicos ayuda a mejorar la planificación regional para la gestión ambiental (Bryan \& Crossman, 2008). Adicionalmente, el mapeo de los SE también puede ayudar a pronosticar los cambios y la necesidad social de servicios ecosistémicos en escenarios futuros alternativos de cambio demográfico y de uso de la tierra (Daily, 2000).

\section{Descripción del área de estudio}

Aruba es una pequeña isla en el archipiélago caribeño, cerca de la costa venezolana. La isla era anteriormente parte de las Antillas Neerlandesas, pero en 1986 se convirtió en una parte autónoma separada del Reino de los Países Bajos (Hoetink, 2021). La mayoría de la población de Aruba es étnicamente mixta - con cepas de holandés, amerindio, africano y español - (Hoetink, 2021). Uno de los pilares principales de la economía de Aruba es el turismo, ya que los viajes y el turismo proporcionan empleo e ingresos a los ciudadanos de Aruba. En 2014, la contribución total de los viajes y el turismo fue equivalente al 88,4 por ciento del PBI de la isla y generó el 91,2 por ciento del empleo total (Turnern, 2015).

El turismo depende de los ecosistemas costeros, marinos y terrestres. Los turistas se sienten atraídos a Aruba debido a las playas de arena blanca, los arrecifes de coral, la costa escarpada y el entorno desértico. Las principales actividades turísticas son ir a las playas, buceo y snorkel, turismo, etc. Playas como "Palm Beach" y "Eagle Beach", que son las más cercanas a los hoteles, son a menudo visitadas por los turistas. Aruba también ofrece lugares atractivos para bucear donde se pueden ver arrecifes de coral. Otra de las principales atracciones de la costa son las dunas de arena, siendo las más famosas las llamadas "Dunas de California". Aruba también ofrece un ambiente terrestre muy atractivo con montañas y formaciones rocosas. La isla tiene algunas colinas aisladas de lado empinado que hacen hermosos paisajes. Una de las montañas más populares es Hooiberg. Algunas de las formaciones rocosas más famosas son la formación rocosa Cassibari y la formación rocosa Ayo. Además, Aruba tiene un parque nacional llamado Parque Nacional Arikok. El parque es conocido por diferentes variedades geológicas como formaciones de lava y formaciones rocosas (Parque Nacional Aruba). 
Sin embargo, estas atracciones turísticas se están viendo afectadas por la degradación ambiental. Entre las principales causas de la degradación de los ecosistemas se encuentran el mal manejo de residuos sólidos, la acidificación de los océanos que genera la pérdida de arrecifes de coral y la urbanización que causa pérdida de biodiversidad y cambio de uso de suelos (Derix, 2014). Estos factores pueden afectar cada vez más la capacidad de Aruba de proporcionar SE estéticos y recreativos. Por ejemplo, a menos que se tomen alguna medida, el inadecuado manejo de residuos sólidos afectará al turismo en las playas y la pérdida de los arrecifes de coral y biodiversidad marina afectará al buceo recreativo. Adicionalmente, el gobierno de Aruba está comprometido con la implementación de los Objetivos de Desarrollo Sostenible (ODS) de las Naciones Unidas. Dentro del objetivo 14 se encuentra, conservar al menos el $10 \%$ de la costa y áreas marinas, de acuerdo con las normas nacionales y derecho internacional (Indicator Working Group, 2018). En el año 2018, se está conservando el 0,3\% de las áreas protegidas (Indicator Working Group, 2018).

\section{Diseño de la encuesta}

Este estudio utilizó los datos sistema de información geográfica de mapeo participativo obtenidos de una encuesta realizada a los turistas antes de salir de Aruba para determinar los "hotpots" culturales más importantes (apreciación estética y recreativa). La encuesta fue diseñada por Wolfs Company en estrecha colaboración con Yabi Consultancy y el Departamento de Naturaleza y Medio Ambiente de Aruba. Se pidió a los turistas que señalaran en un mapa turístico de papel los lugares más importantes con valor estético y recreativo. Los encuestados utilizaron marcadores de color (dos $\mathrm{mm}$ ) para dibujar símbolos indicando los tipos de actividades/valores estéticos y recreativos. Se pidió a los encuestados que utilicen puntos, ya que son más adecuados que los polígonos para estudios a gran escala como este (Brown \& Kyttä, 2014). Los valores recreativos fueron obtenidos pidiendo a los encuestados que señalen los 4 lugares más importantes donde realizaron actividades terrestres y marinas. Similarmente, los valores estéticos fueron obtenidos pidiendo a los turistas que indiquen los 3 lugares con más hermosos paisajes. Para facilitar la comprensión de las actividades terrestres (actividades terrestres y actividades marinas y costeras) y los valores estéticos, los términos se explicaron como se muestra en la Tabla 1.

Tabla 1. Explicaciones de los valores

\begin{tabular}{ll}
\hline \multicolumn{1}{c}{ Tipo de valor } & \multicolumn{1}{c}{ Explicación } \\
\hline $\begin{array}{l}\text { Actividades } \\
\text { terrestres }\end{array}$ & $\begin{array}{l}\text { Ciclismo, Caminar, Correr, } \\
\text { Senderismo, Camping, y } \\
\text { Observación de aves / vida } \\
\text { silvestre, Conducción Jeep / Tour }\end{array}$ \\
$\begin{array}{l}\text { Actividades } \\
\text { marinas y } \\
\text { costeras }\end{array}$ & $\begin{array}{l}\text { Pesca, Ir a la playa, Natación, } \\
\text { / vela / kayak, y Kiting / windsurf, }\end{array}$ \\
& Paseos a caballo \\
Estético & $\begin{array}{l}\text { Valoro estos lugares debido a los } \\
\text { hermosos paisajes (Brown, 2005) }\end{array}$ \\
\hline
\end{tabular}

\section{Muestra}

La muestra se recogió utilizando una encuesta presencial realizada antes de que los turistas se vayan de Aruba. Este tipo de encuesta se utilizó porque los turistas expresan y reflejan mejor sus puntos de vista una vez que han obtenido los beneficios de los servicios ecosistémicos. Esto también disminuye los sesgos; debido a que los encuestados están familiarizados con los servicios ecosistémicos (Daly et al., 2015). Dado que la mayoría de los turistas hablan inglés, la encuesta se llevó a cabo en este idioma.

Aruba tiene dos tipos de turistas: turista de estancia (el viaje incluye una estancia de una noche) y turista de crucero (el viaje no incluye una estancia de una noche). No se conoce exactamente el tamaño de la población de los turistas. Se sabe que, en el año 2014, Aruba recibió 1072082 turistas de estancia y 667095 turistas de crucero (CBS, 2014). Adicionalmente, las características de la población de turistas van cambiando cada año.

Cómo no se sabe el tamaño exacto de la población de turistas, se utilizó la fórmula para calcular la muestra en poblaciones infinitas (Peacock \& Peacock, 2011; Rumsey, 2016): 


$$
n=\frac{Z_{a}^{2} * p *(1-p)}{d^{2}}
$$

Donde:

$\mathrm{n}=$ Tamaño de la muestra.

Za 2 = Es un parámetro que depende del intervalo de confianza. En este caso, 1,962 (ya que la seguridad es del 95\%).

$\mathrm{p}=$ La probabilidad o proporción de que ocurra el evento estudiado. La proporción no necesita ser un valor muy preciso. Si el investigador no conoce el valor de p, se debe utilizar 0,5 ( (Peacock \& Peacock, 2011). En este caso $50 \%=0,5$.

$\mathrm{q}=$ Probabilidad de que no ocurra el evento estudiado= $1-\mathrm{p}$ (en este caso $1-0,5=0,5$ )

$\mathrm{d}=$ error de estimación máximo aceptado (en este caso deseamos un $6 \%$ ).

Reemplazando los valores se obtuvo:

$$
n=\frac{1,96^{2} * 0,5 *(1-0,5)}{0,06^{2}}=266,77=267
$$

Para el caso de los turistas de crucero, se tomó una muestra aleatoria de 269 turistas de cruceros, debido a que una muestra aleatoria elimina el sesgo en el proceso de muestreo (Rumsey, 2016). Para el caso de los turistas de estancia, se tomó una muestra aleatoria estratificada de acuerdo con la nacionalidad de 316 turistas de estancia. Las nacionalidades de los turistas de estancia se obtuvieron de la Oficina Central de Estadísticas (CBS, 2014).

Los datos fueron recopilados por un equipo de entrevistadores de la Universidad de Aruba, los cuales recibieron la misma capacitación para evitar sesgos en las respuestas. Los datos se recopilaron durante los meses de octubre de 2016, noviembre de 2016 y marzo de 2017. Los lugares seleccionados para llevar a cabo la encuesta fueron el aeropuerto y el puerto, es decir, los lugares donde los turistas salen de Aruba. Además, hubo una variación en los horarios de las encuestas con el fin de tener aleatoriedad en el proceso de muestreo.

\section{Representatividad de la muestra}

Las características de la población de turistas cambian cada año. De igual forma, se verificó la representatividad de la muestra de turistas de estancias utilizando un perfil demográfico real de los turistas de Estancia que visitan Aruba. El perfil demográfico real de turistas de Estancia que visitan Aruba se obtuvo del Tourist Profile - Year Report 2013 (CBS, 2013). Los datos Tourist Profile - Year Report 2013 (CBS, 2013) no contienen información de género y por lo tanto los datos de género se obtuvieron del (World Bank, 2017). Por otro lado, no fue posible saber si la muestra de turistas de Estancia es representativa en términos de ingresos porque el 50 por ciento de los turistas de Estancia dijeron que preferían no responder preguntas sobre su nivel de ingresos. Se utilizaron las variables de género, nacionalidad y la edad para determinar la representatividad de la muestra de los turistas de estancia. Se utilizó la prueba de chi - cuadrada no paramétrica en SPSS (V 22) para calcular si los datos obtenidos son estadísticamente representativos.

No fue posible determinar si la muestra de los turistas de crucero era representativa, porque no hay información actualizada disponible del perfil de estos turistas. El último perfil de turistas de cruceros se remonta a 2002 (CBS, 2003).

Se obtuvieron los siguientes resultados sobre la representatividad de la muestra de turistas. La proporción de género de los turistas del crucero es similar a la proporción de género del mundo $\left(\mathrm{x}^{2}\right.$ $(1)=1,393, p=0,238)$. La muestra de turistas de estancia es representativa para el género $\left(\mathrm{x}^{2} \quad(1)\right.$ $=0,162, \mathrm{p}=0,687)$ y no es representativa para la distribución por edades $\left(\mathrm{x}^{2}(5)=99,447, \mathrm{p}=0,000\right)$ (Tabla 2). Además, la muestra de los turistas de estancia tiene una sobrerrepresentación de personas con nacionalidades de países del Caribe (Figura 2). Las demás nacionalidades están bien representadas en la muestra (Figura 2). La muestra de turistas de estancia muestra una sobrerrepresentación de los turistas con edades entre 20 y 29 años, así como una subrepresentación de los turistas con edades entre 40 y 49 años (Figura 2). Para corregir estas subrepresentaciones y sobre-representaciones se tendría que eliminar datos del grupo sobre representado (edades entre 20 - 29 años) y doblar los datos del grupo subrepresentado (40-49 años). Sin embargo, se decidió no realizar esta corrección porque la eliminación o adición de datos de la muestra podría generar sesgos o tergiversar otras características demográficas. 
Tabla 2. Resultados de la prueba de chi-cuadrado de los turistas de crucero y de estancia.

\begin{tabular}{lcccc}
\hline $\begin{array}{l}\text { Características } \\
\text { demográficas }\end{array}$ & \multicolumn{2}{c}{$\begin{array}{c}\text { Turistas de } \\
\text { crucero }\end{array}$} & \multicolumn{3}{c}{$\begin{array}{c}\text { Turistas de } \\
\text { estancia }\end{array}$} \\
\cline { 2 - 5 } & $\begin{array}{c}\text { Chi- } \\
\text { cuadrado }\end{array}$ & $\mathrm{p}$ & $\begin{array}{c}\text { Chi- } \\
\text { cuadrado }\end{array}$ & $\mathrm{p}$ \\
\hline Genero & 1.39 & 0.24 & 0.16 & 0.68 \\
Edad & & & 99.45 & 0.00 \\
\hline
\end{tabular}

En la Tabla 3 se muestran las características demográficas de los turistas encuestados antes de salir de Aruba. El porcentaje de turistas crucero y turistas de estancia fue de $46 \%$ y $54 \%$ respectivamente. Tanto las opiniones femeninas como las masculinas están bien representadas en la muestra. La mayoría de los encuestados provienen de los Estados Unidos y Canadá. De igual forma, la mayoría de los turistas tienen un alto nivel de educación. La muestra de turistas de estancia tiene un porcentaje mayor de turistas de América Latina que la muestra de cruceros. Los resultados del análisis del sistema de información geográfica de mapeo participativo corresponden a una población con las características demográficas de la muestra.

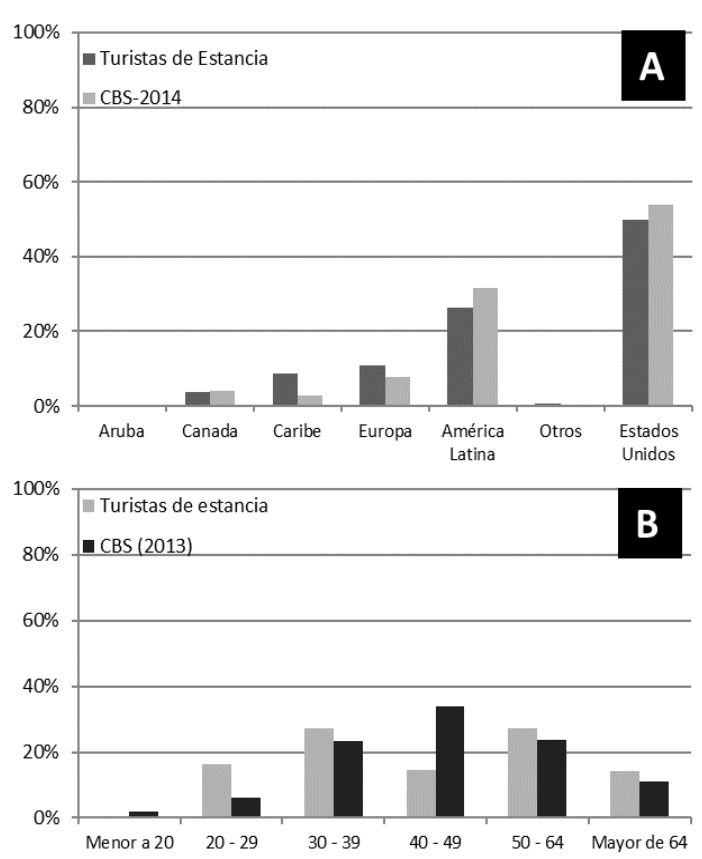

Figura 2. Distribución de los turistas de estancia por nacionalidad (A) y edad (B).

Tabla 3. Resumen de las características demográficas.

\begin{tabular}{|c|c|c|c|c|c|c|c|}
\hline \multirow[t]{2}{*}{ Variables } & & \multicolumn{2}{|c|}{ Turistas de crucero $(n=269)$} & \multicolumn{4}{|c|}{ Turistas de estancia $(n=316)$} \\
\hline & & \# desaparecidos & Frecuencia & $\%$ & \# desaparecidos & Frecuencia & $\%$ \\
\hline Género & masculino & 1 & 145 & 53,9 & 0 & 156 & 49,4 \\
\hline \multirow[t]{3}{*}{ Nacionalidades } & Europa & 1 & 41 & 12,7 & 0 & 61 & 10,8 \\
\hline & USA y Canadá & & 204 & 75,8 & & 169 & 53,5 \\
\hline & Latinoamérica & & 11 & 4,1 & & 83 & 26,3 \\
\hline Educación & Universitario & 0 & 181 & 67,3 & 0 & 228 & 72,2 \\
\hline
\end{tabular}

\section{Ejercicio de mapeo participativo}

Para determinar los lugares donde se experimentan los valores más altos de recreación y apreciación estética, se convirtieron los mapas de sistema de información geográfica de mapeo participativo en mapas de "puntos críticos". Para este estudio, se considera un punto crítico una zona con abundancia de eventos. Un punto crítico recreativo se define como una zona donde la mayoría de encuestados indicó que realizó una actividad recreativa y un punto crítico estético sería un lugar donde la mayoría de encuestados indicó que hay un hermoso paisaje.
La información se procesó en el software ArcGIS (V 9.4). Se recibieron un total de 527 mapas en papel de las 584 encuestas realizadas. Cada mapa fue digitalizado y codificado utilizando el Sistema Geodésico Mundial de 1984 (Datum: WGS 1984). Luego, el Sistema de Coordenadas Geográficas fue proyectado a WGS 1984 UTM Zona 19 N, que es la mejor proyección para Aruba. El área de interés era un colchón de 1,000 m alrededor de la costa de la isla. 
Se utilizaron un total de 352 mapas válidos para mapear los puntos críticos recreativos (los lugares donde la mayoría de encuestados indicó que realizó una actividad recreativa) y, de manera similar, se utilizaron 258 mapas válidos para mapear los puntos críticos estéticos (lugares donde la mayoría de encuestados indicó que hay un hermoso paisaje).

Se utilizó la función de densidad de puntos con tamaño de celda 90 y se seleccionó la clasificación según los umbrales naturales de Jenks para obtener nueve clases de nivel de densidad. De las nueve clases, sólo las tres clases superiores fueron consideradas como puntos críticos. Se utilizó la clasificación de umbrales naturales de Jenks porque destaca las diferencias entre las diferentes clases (Burkhard \& Maes, 2017).

Luego se creó un mapa de las áreas naturales significativas de la isla en función de la densidad de los puntos estéticos y recreativos. El mapa de este análisis se obtuvo de (Bubalo, 2016), se basó en tres fuentes: las ideas de un ejercicio de grupo de enfoque llevado a cabo para definir las áreas naturales importantes, dos zonas designadas para la conservación futura en el plan espacial de Aruba e información sobre las zonas del Parque Natural de Arikok. Al final, el mapa de (Bubalo, 2016) consideró 13 áreas naturales. Usando la función de unir datos para combinar el conjunto de datos de SE culturales de los turistas y el mapa de las áreas naturales y luego clasificamos las áreas de acuerdo con el número de puntos por kilómetro cuadrado.

\section{Comparación de sistema de información geográfica de mapeo participativo entre partes interesadas}

Los mapas sistema de información geográfica de mapeo participativo de los turistas se compararon con los mapas de los ciudadanos locales (Bubalo, 2016) para determinar si existe una diferencia entre los puntos críticos elegidos por los turistas y por los ciudadanos locales. Del mismo modo, los mapas sistema de información geográfica de mapeo participativo de los turistas de Crucero se compararon con los mapas sistema de información geográfica de mapeo participativo de turistas de estancia para determinar si el tipo de turista afecta al patrón de puntos críticos. Estudios anteriores han demostrado que las motivaciones y las características demográficas influyen en las preferencias de lugares con altos valores de apreciación de los SE culturales (Ribeiro \& Katia, 2016, Plieninger et al., 2013; Van Zanten et al., 2014).

Los datos se perdieron al vincular los mapas de mapeo participativo con el tipo de turistas. Como consecuencia, se utilizaron 107 mapas válidos para mapear el valor estético de los turistas de Crucero y 114 mapas válidos se utilizaron para mapear el valor estético de los turistas de estancia. Del mismo modo, se utilizaron 135 mapas para mapear el valor recreativo de los turistas de crucero y 158 mapas válidos se utilizaron para mapear el valor recreativo de los turistas de estancia.

Tabla 4. Valores para los mapas de comparación del mapeo participativo.

\begin{tabular}{|c|c|c|}
\hline Valor & Denominación & Significado \\
\hline 0 & Acuerdo bajo & $\begin{array}{l}\text { Las dos partes interesadas } \\
\text { indican que la zona es un } \\
\text { punto frío (zona con escasez } \\
\text { de eventos). }\end{array}$ \\
\hline 1 & $\begin{array}{l}\text { Un valor } \\
\text { intermedio }\end{array}$ & $\begin{array}{l}\text { Una parte interesada indica } \\
\text { que la zona es neutral y la } \\
\text { otra indica que en un punto } \\
\text { frío (zona con escasez de } \\
\text { eventos). }\end{array}$ \\
\hline 2 & $\begin{array}{l}\text { Sin valores } \\
\text { extremos }\end{array}$ & $\begin{array}{l}\text { Las dos partes interesadas } \\
\text { indican que es una zona } \\
\text { neutral. }\end{array}$ \\
\hline 10 & $\begin{array}{l}\text { En desacuerdo } \\
\text { acerca de los } \\
\text { valores bajo y } \\
\text { valores }\end{array}$ & $\begin{array}{l}\text { Una parte interesada indica } \\
\text { que la zona es un punto } \\
\text { crítico (zona con abundancia } \\
\text { de eventos) y la otra indica } \\
\text { que es un punto frío (zona } \\
\text { con escasez de eventos). }\end{array}$ \\
\hline 11 & Un valor alto & $\begin{array}{l}\text { Una parte interesada indica } \\
\text { que la zona es un punto } \\
\text { crítico (zona con abundancia } \\
\text { de eventos) t y la otra que es } \\
\text { una zona neutral. }\end{array}$ \\
\hline 20 & Acuerdo alto & $\begin{array}{l}\text { Las dos partes interesadas } \\
\text { indican que la zona es un } \mathrm{h} \\
\text { punto crítico (zona con } \\
\text { abundancia de eventos) }\end{array}$ \\
\hline
\end{tabular}

Para ambas comparaciones, las nueve clases de los dos conjuntos de datos (datos de los turistas y datos de los ciudadanos locales) se reclasificaron en puntos críticos (una zona con abundancia de eventos 
recreativos o de apreciación estética), puntos fríos (una zona con escasez de eventos recreativos o de apreciación estética) y áreas neutrales. Las tres clases de valor más altos se clasificaron como puntos críticos, asignándoles el valor de 10; las tres de las clases de valor más bajo se clasificaron como puntos fríos y se les dio el valor de cero y finalmente las tres clases en el medio se clasificaron como neutras y se les asignó el valor de uno. A continuación, se superpusieron los mapas de los puntos críticos de los diferentes datasets (turistas y ciudadanos locales) utilizando la función "Agregar" de Calculadora ráster para producir mapas de acuerdo que contienen los nuevos valores $(0,1,2,10,11$ y 20$)$ que indican la correspondencia espacial de los dos datasets (Tabla 4).

\section{RESULTADOS Y DISCUSIÓN}

\section{Distribución espacial de los servicios de los ecosistemas culturales}

La Figura 3 muestra los resultados del análisis de densidad de los datos del sistema de información geográfica de mapeo participativo para el valor estético y el valor recreativo para los turistas de Aruba. En total, se utilizaron 865 puntos como indicadores del valor recreativo y 375 puntos como indicadores del valor estético, lo que indica que los valores recreativos se marcaron con mayor frecuencia que los valores estéticos.

Las zonas con los valores estéticos y recreativos más elevados se encuentran principalmente a lo largo de la costa. Estos resultados concuerdan con los hallazgos de estudios anteriores que muestran que hay un efecto positivo del agua en la apreciación estética (García-Llorente et al., 2012; Pastur et al., 2016, Plieninger et al., 2013) y la recreación (Termansen et al., 2004; Plieninger et al., 2013). El punto estético más importante es Eagle Beach, seguido de Palm Beach y California Light House. La zona con mayor valor recreativo es Eagle Beach, seguida de Palm Beach. Es importante plantear estrategias de protección de Eagle Beach, Palm Beach y California Light House ya que son muy apreciadas por los turistas. Las actividades costeras y marinas más frecuentes son nadar/vadear e ir a la playa (el 48,05 y el 40,67 \% de las respuestas de los encuestados fueron nadar/vadear e ir a la playa, respectivamente). La actividad terrestre más frecuente es caminar (el 77,93\% de los encuestados respondieron que su principal actividad era caminar).

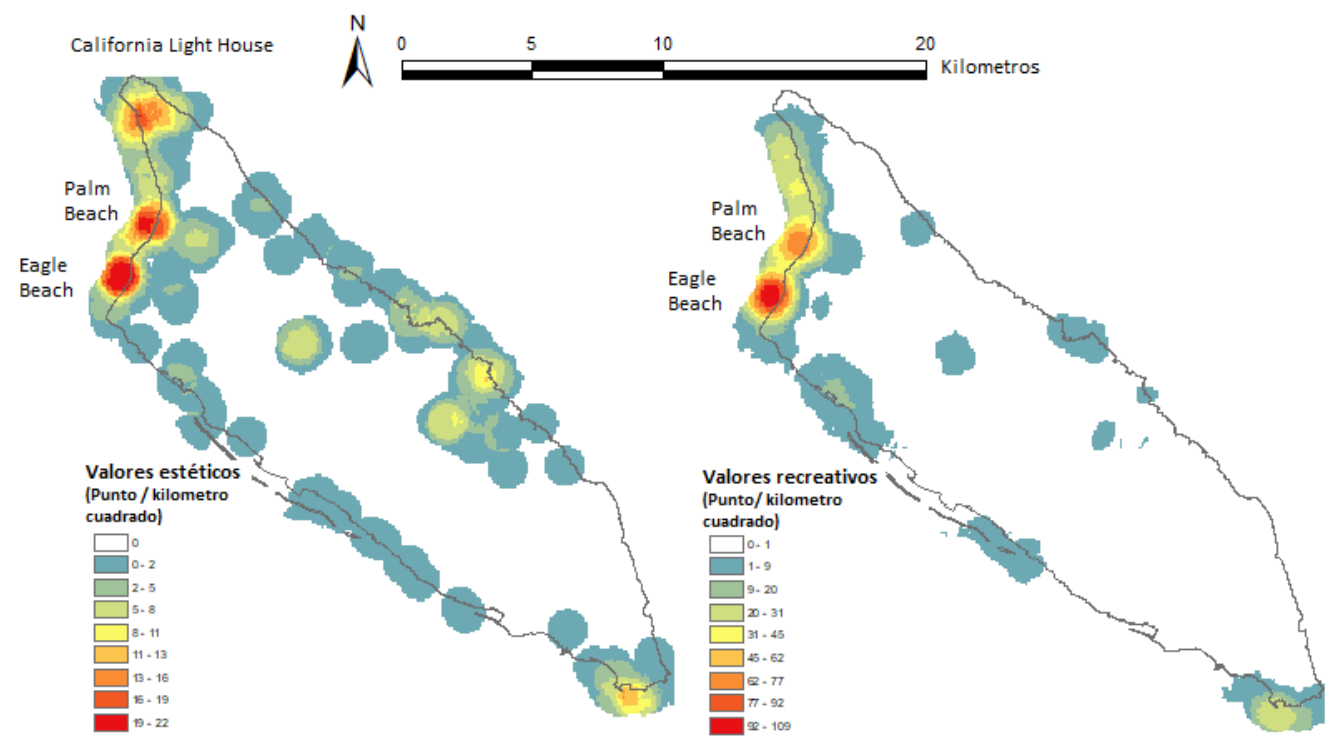

Figura 3. Densidad de puntos de valor estético (izquierda) y de valor recreativo (derecha).

El Parque Nacional de Arikok está situado en la parte nororiental de Aruba. Aunque no es un punto crítico (una zona con abundancia de eventos recreativos o de apreciación estética), el Parque Nacional de Arikok fue clasificado mejor en términos de apreciación estética que en términos de 
actividad recreativa. La apreciación estética suele estar relacionada con los parques nacionales (Pastur et al., 2016), lo que podría ser una explicación potencial de los valores más altos.

El 56\% del conjunto de datos referentes a los valores estéticos y recreativos estaba situado en áreas naturales identificadas en el mapa de áreas naturales realizado por (Bubalo, 2016). Como se refleja en la Figura 4, los niveles de densidad más altos se encuentran en la Formación de Rocosa Casibari, las White Beaches (Oeste) y la Formación Rocosa Ayo. El análisis sistema de información geográfica de mapeo participativo realizado por (Bubalo, 2016) también indica que White Beach (Oeste) es una de las áreas naturales con puntos de alta densidad cultural, pero no está de acuerdo en las otras ubicaciones de puntos de alta densidad. En contraste con nuestros hallazgos, (Bubalo, 2016) también identifica Hooiberg y Manglar como áreas naturales con alta densidad y no considera a la Formación Rocosa Casibari y a la Formación Rocosa Ayo como áreas naturales con alta densidad. Es importante resaltar que el análisis del sistema de información geográfica de mapeo participativo (Bubalo, 2016) utiliza las opiniones de los ciudadanos locales y este estudio utiliza las opiniones de los turistas. Además, esta comparación revela que la zona White Beaches en el oeste tiene altos valores estéticos y recreativos tanto para los turistas como para los ciudadanos locales. Finalmente, es importante señalar que las formaciones rocosas Casibari y Ayo tienen un alto nivel de densidad porque hay varios puntos en áreas pequeñas $(0,2599$ kilómetros cuadrados y 0,3899 kilómetros cuadrados respectivamente). Otras áreas naturales, como Conservation Zone (Norte), tienen un alto número de puntos, pero en un área extendida y por lo tanto tienen una baja densidad.

\section{Comparación de sistema de información geográfica de mapeo participativo entre partes interesadas \\ Comparación entre turistas y ciudadanos locales.}

Para comparar los resultados de los turistas con los residentes locales, se superpusieron los mapas de los turistas y se contrastaron con el mapeo participativo de los ciudadanos locales (Bubalo, 2016) (Figura 5).
El área de alto acuerdo para el valor estético es California Light House, indicando que esta zona es un punto crítico estético para los turistas y residentes locales (la mayoría de encuestados indicó que hay un hermoso paisaje). Las áreas en desacuerdo para los valores estéticos son Palm Beach y el Parque Nacional Arikok. Los turistas consideran Palm Beach un lugar con alta apreciación estética, mientras que los ciudadanos locales no lo hacen. Por otro lado, los ciudadanos locales piensan que el Parque Nacional Arikok es un punto con altos valores de apreciación estética, mientras que los turistas no lo piensan. Eagle Beach está clasificada como un área mixta, que contiene zonas de desacuerdo y una zona con un valor alto en apreciación estética. En la zona de desacuerdo, los turistas tienen una alta apreciación estética por el agua de Eagle Beach y los ciudadanos locales no. En la zona con un valor alto estético, los ciudadanos locales consideran el continente de Eagle Beach una zona estética neutra, mientras que los turistas lo consideran un punto con altos valores de apreciación estética. Esto explica los valores mixtos que se encuentran en Eagle Beach.

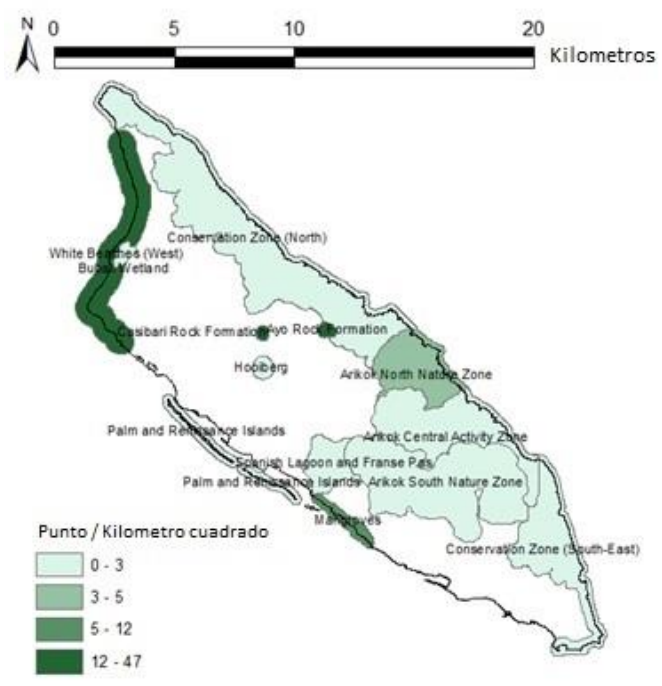

Figura 4. Densidad de puntos de valores estéticos y recreativos en 13 áreas naturales (Bubalo, 2016).

Los ciudadanos locales señalaron mayor cantidad de zonas con altor valor recreativo que los turistas. Los ciudadanos locales identificaron 5 zonas con alto valor recreativo y los turistas identificaron 2 zonas 
con altos valores recreativos. La zona de alto acuerdo en cuanto al valor recreativo es Eagle Beach, indicando que es un punto crítico recreativo para los turistas y residentes locales (la mayoría de encuestados indicó que realizó una actividad recreativa). La zona de desacuerdo en cuanto al valor recreativo es Surfside Beach, lo que indicaría que los ciudadanos locales disfrutan de actividades recreativas en Surfside Beach mientras que los turistas no. Además, las áreas de un valor alto (una parte interesada indica que la zona es un punto crítico y la otra que es una zona neutral) para el valor recreativo son Palm Beach, Arashi Beach, Boca Catalina Beach y Malmok Beach. Los turistas consideran que Palm Beach es un punto de interés recreativo y los residentes locales piensan que es una zona neutral. Por otra parte, los residentes locales consideran que Arashi Beach, Boca Catalina Beach y Malmok Beach son puntos de atracción para la recreación, mientras que los turistas consideran las playas como zonas neutrales.

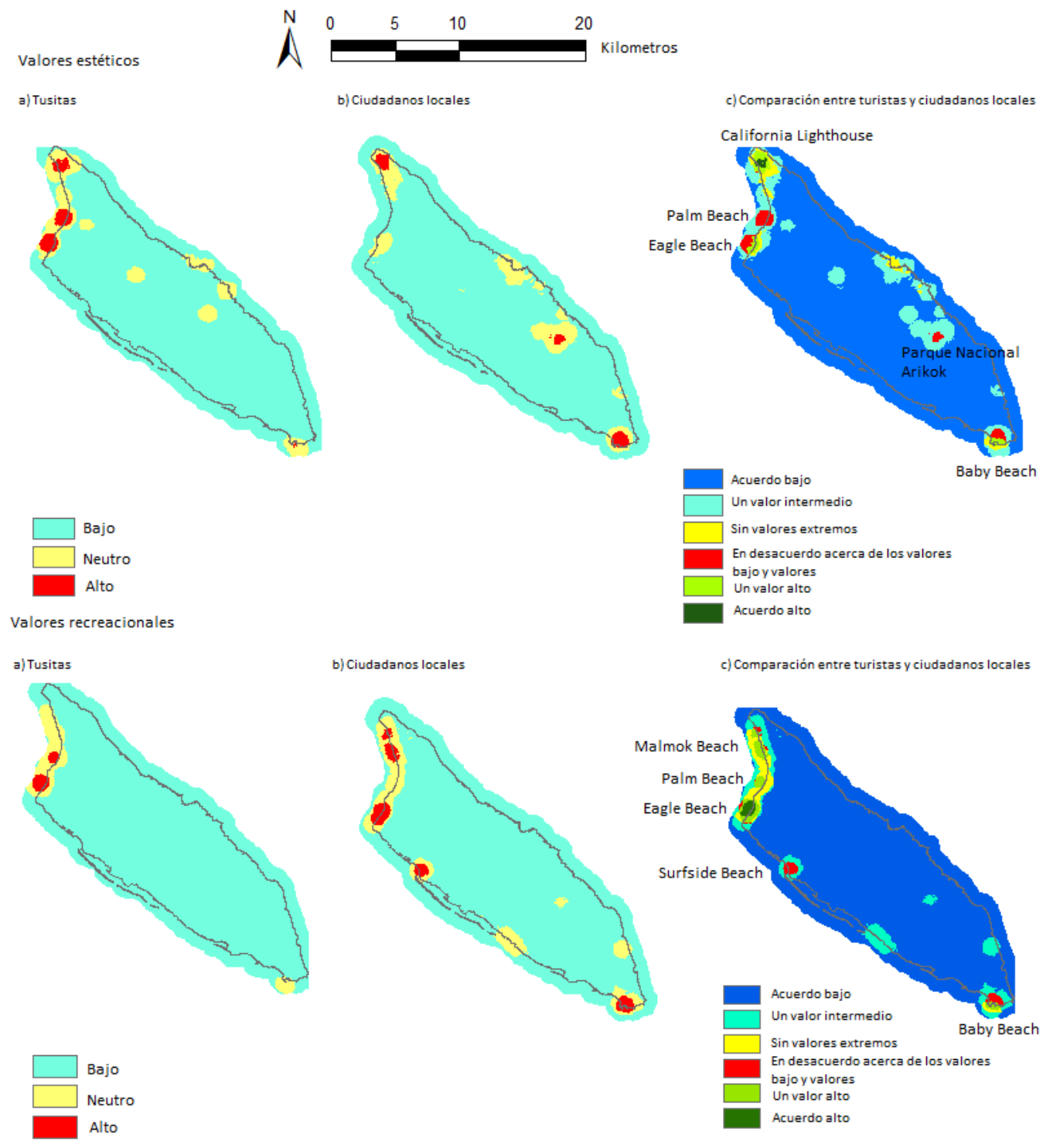

Figura 5. Mapas que comparan los valores estéticos (arriba) y los valores recreativos (abajo) seleccionados por turistas y ciudadanos locales. 
Baby Beach es un lugar estético y recreativo para los ciudadanos locales y los valores culturales se concentran en los paisajes. Para los turistas, sin embargo, es una zona neutral y los valores culturales se concentran en el océano. Esta zona también puede clasificarse como zona mixta, ya que contiene un área de desacuerdo y un valor alto para los valores estéticos y recreativos.

Los ciudadanos locales y los turistas tienen diferentes motivaciones para seleccionar los puntos de atracción (Ribeiro \& Katia, 2016; Plieninger et al., 2013). Un factor que puede explicar algunas diferencias es que los ciudadanos locales tienen un alto sentido de pertenencia e identificación porque nacieron y se criaron en Aruba (Ribeiro \& Katia, 2016). Algunos aspectos culturales (por ejemplo, el conocimiento de algunas tradiciones y leyendas) también podrían desempeñar un papel en zonas específicas percibidas por los ciudadanos locales y no percibidas por los turistas (Ribeiro \& Katia, 2016).
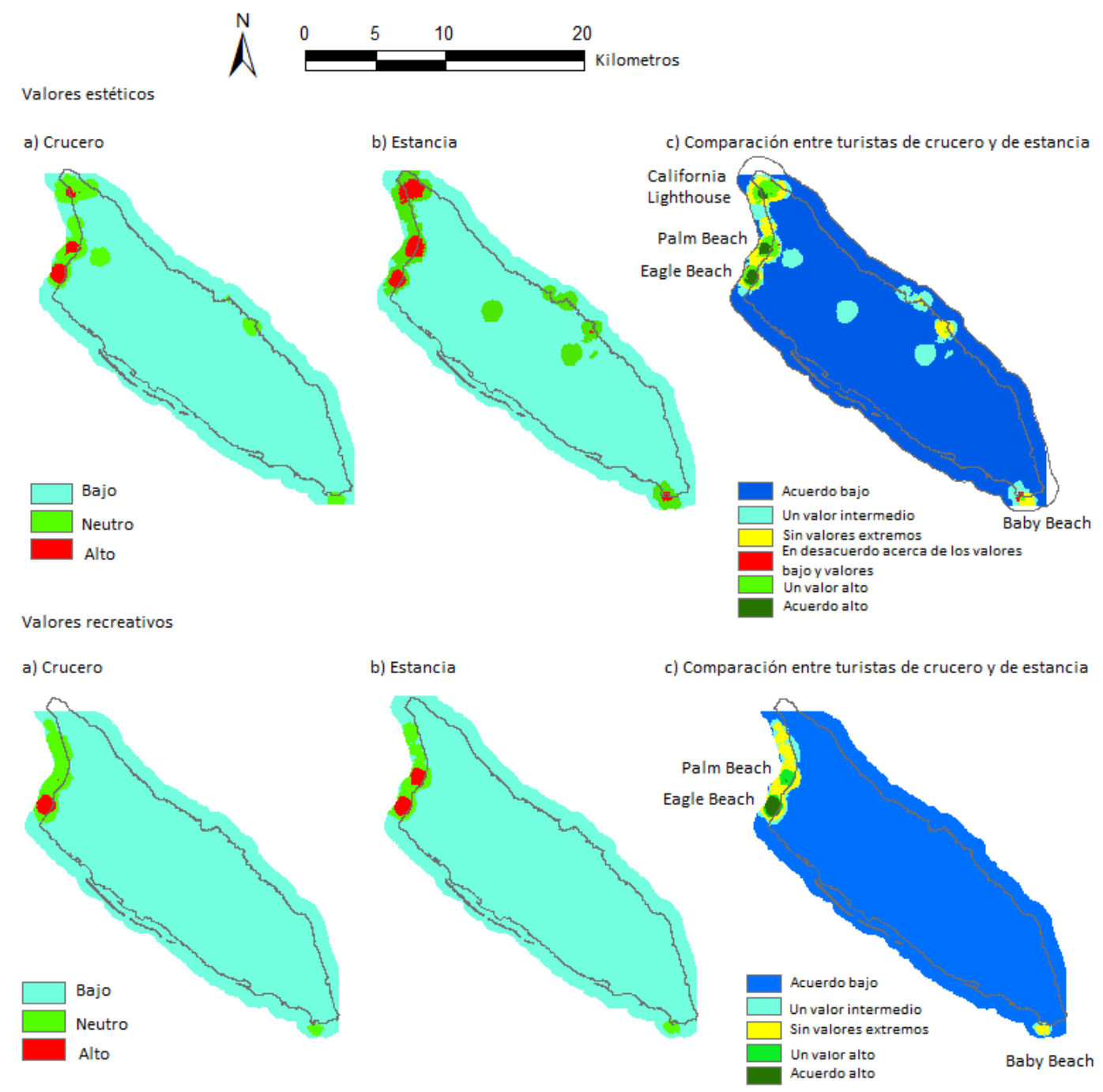

Figura 6. Mapas que comparan los valores estéticos (arriba) y los valores recreativos (abajo) seleccionados por turistas de estancia y turistas de crucero.

Por otra parte, los turistas podrían estar motivados por su interés en la naturaleza y su sentimiento de placer (Ribeiro \& Katia, 2016). Variables como la edad, el sexo, el empleo y la frecuencia con que se 
visitan algunas zonas del paisaje también podrían influir en las zonas que se perciben como puntos críticos (Plieninger et al., 2013). Adicionalmente, para los ciudadanos locales las actividades recreativas no se planifican y no están asociadas con el gasto de dinero y para los turistas, estas se planifican y están asociadas con el gasto económico (Chakraborty et al., 2020).

\section{Comparación entre los turistas de crucero y los turistas de estancia}

Se compararon los mapas participativos de los turistas del crucero con los mapas participativos de los turistas de estancia (Figura 6).

Eagle Beach fue un área de acuerdo alto para los valores estéticos y recreativos, confirmando que este es un punto crítico estético y recreativo para los turistas de estancia y turistas de crucero (la mayoría de encuestados indicó que realizó una actividad recreativa e indicó que hay un hermoso paisaje).

Adicionalmente, las áreas de acuerdo alto en cuanto a los valores estéticos son Palm Beach y California Light House, confirmando que en estas zonas se aprecian hermosos paisajes. Se encontraron pocas zonas en desacuerdo para la apreciación estética. Además, los turistas de estancia consideran que Baby Beach es un punto con altos valores de apreciación estética mientras que los turistas de los cruceros consideran a esta playa como una zona neutral. Adicionalmente, los turistas de estancia tienen una mayor apreciación estética del Parque Nacional de Arikok que los turistas de crucero. La diferencia principal entre ambos turistas es que los turistas de crucero están más limitados por el tiempo que pasan en Aruba (un día). Por lo tanto, son más críticos en la selección de los lugares que visitan y prefieren visitar los lugares cercanos. Como resultado, es más probable que más turistas de estancia visiten el Parque Nacional de Arikok (que es una actividad que requiere tiempo), lo que explicaría la mayor apreciación estética de las estancias en el Parque Nacional de Arikok. No se encontraron zonas en desacuerdo para los valores recreativos.

Palm Beach fue una zona con un alto valor recreativo. Los turistas de estancia consideran a
Palm Beach como punto de interés recreativo, mientras que los turistas de cruceros consideran a Palm Beach como un área recreativa neutral.

\section{CONCLUSIÓN}

Utilizando los datos de mapeo participativo de los turistas se identificó los lugares donde se experimentan mayor satisfacción de los servicios culturales (recreación y apreciación estética) en Aruba. La investigación demuestra la importancia de Eagle Beach, Palm Beach y California Lighthouse para los turistas, ya que Eagle Beach y Palm Beach son considerados puntos de interés estéticos y recreativos (la mayoría de encuestados indicó que realizó una actividad recreativa o hay un hermoso paisaje); y California Lighthouse es considerado una zona con alta apreciación estética (la mayoría de encuestados indicó que hay un hermoso paisaje). Este lugar, situado en el norte de la isla, se encuentra dentro de la zona de conservación de la naturaleza definida por el Plan Espacial de 2009, pero hasta el día de hoy carece de protección. Comparando los datos de mapeo participativo de los turistas con los datos de mapeo participativo de los ciudadanos locales, se identificaron que hay similitudes y diferencias en la identificación de puntos de interés recreativo y de apreciación estética en Aruba. Entre las similitudes, los turistas y ciudadanos locales consideran a California Light House un punto crítico estético (la mayoría de encuestados indicó que hay un hermoso paisaje) y a Eagle Beach un punto crítico recreativo (la mayoría de encuestados indicó que realizó una actividad recreativa). Entre las diferencias, se encontraron como zonas de desacuerdo entre ambas partes interesadas (una parte interesada indica que la zona es un punto crítico y la otra indica que es un punto frío) a Palm Beach, el Parque Nacional Arikok y Surfside Beach. Por otro lado, se encontrarán más similitudes que diferencias entre los turistas de estancia y cruceros. Los lugares de mayor apreciación cultural común para los turistas de estancia y crucero son Eagle Beach, Palm Beach y California Lighthouse y se encontraron pocas zonas en desacuerdo para la apreciación estética y no se encontraron zonas en desacuerdo para los valores recreativos. Esta investigación contribuye a identificar diferentes pautas en el uso de los 
servicios de los ecosistemas culturales de los turistas y la información es útil para la toma de decisiones informadas respecto a la conservación y el uso adecuado de suelo. La investigación, ha mapeado los servicios ecosistémicos culturales de apreciación estética y recreativos. Sin embargo, se puede utilizar una metodología similar para mapear otros servicios ecosistemas culturales como los espirituales y educacionales.

\section{Conflictos de intereses}

Los autores firmantes del presente trabajo de investigación declaran no tener ningún potencial conflicto de interés personal o económico con otras personas $\mathrm{u}$ organizaciones que puedan influir indebidamente con el presente manuscrito.

\section{Contribuciones de los autores}

Preparación y ejecución: VSF; Desarrollo de la metodología: VSF; Concepción y diseño: VSF; Edición del artículo: VSF; Supervisión del estudio: VSF.

\section{LITERATURA CITADA}

- Assessment, M. E. (2006). Ecosystems and Human Well-being: A Framework for Assessment. Ecosystems and Their Services. 22pp. Disponible en https://www.millenniumassessment.org/docume nts/document.300.aspx.pdf

- Bown, G., Montag, J., \& Lyon, K. (2012). Public participation GIS: A method for identifying ecosystem services. Society \& Natural Resources, 25(7), 633-651. https://doi.org/10.1080/08941920.2011.621511

- Brown, G. (2005). Mapping spatial attributes in survey research for natural resource management: methods and applications. Society and natural resources, 18(1), 17-39.

- Brown, G., \& Fagerholm, N. (2015). Empirical PPGIS/PGIS mapping of ecosystem services: a review and evaluation. Ecosystem Services, 119133. https://doi.org/10.1016/j,ecoser,2014,10,007

- Brown, G., \& Kyttä, M. (2014). Key issues and research priorities for public participation gIS
(PPGIS): A synthesis based on empirical research. Applied Geography, 46(1), 122-136. https://doi.org/10.1016/j,apgeog.2013.11.004

- Brown, G., \& Reed, P. (2009). Public Participation GIS: a new method for national forest planning. Forest Science, 55(2), 166-182.

- Bryan, B.A., \& Crossman, N.D. (2008). Systematic regional planning for multiple objective natural resource management. Journal of environmental management, 88(4), 11751189. https://doi.org/10.1016/j.jenvman.2007.06.003

- Bubalo, M. (2016). Capturing spatial distribution of cultural ecosystem services in Aruba. Capturing spatial distribution of cultural ecosystem services in Aruba. Amsterdam, Holanda.

- Burkhard, B., \& Maes, J. (2017) Mapping Ecosystem Services. Advanced Books. https://doi.org/10.3897/ab.e12837.

- CBS. [Central Bureau of Statistics]. (2003). Cruise Survey 2002. Disponible en: http://cbs.aw/wp/index.php/2003/10/02/cruissurvey-2002/

- CBS. [Central Bureau of Statistics]. (2013). Tourist Profile-Year Report 2013. Disponible en: $\quad$ http://cbs,aw/wp/wpcontent/uploads/2014/12/TOURISMPROFILE-YEAR-REPORT-2013,pdf

- CBS. [Central Bureau of Statistics]. (2014). Category: General visitors statistics. Disponible en:

http://cbs,aw/wp/index,php/category/tourism/ge neral-visitors-statistics/

- Chakraborty, S., Saha, S.K., \& Selim, S.A. (2020). Recreational services in tourism dominated coastal ecosystems: Bringing the noneconomic values into focus. Journal of Outdoor Recreation and Tourism, 20. https://doi.org/10.1016/j.jort.2020.100279

- Daily, C.G. (2000). Management objectives for the protection of ecosystem services. Environmental Science \& Policy, 3(6), 333-339. https://doi.org/10.1016/S1462-9011(00)00102-7

- Daly, C., Fraser, G., \& Snowball, J. (2015). Willingness to pay for marine-based tourism in the ponta do ouro partial marine reserve, 
mozambique. African Journal of Marine Science, 37(1), 33-40.

- Darvill, R. \& Lindo, Z. (2016). The inclusion of stakeholders and cultural ecosystem services in land management trade-off decisions using an ecosystem services approach. Landscape Ecol 31, 533-545. https://doi.org/10.1007/s10980015-0260-y

- Derix, R. R. (2014). Opportunities and Challenges for Environmental Statistics in Aruba. 11pp. Disponible en: https://cbs.aw/wp/wpcontent/uploads/2015/11/Opportunities-andChallenges-Environmental-Stats-2014.pdf

- García Nieto, A.; Quintas-Soriano, C.; Garcia Llorente, M.; Palomo, I.; Montes, C. \& MartínLópez, B. (2015). Collaborative mapping of ecosystem services: The role of stakeholders' profiles. Ecosystem Services, 13(2), 141-152. https://doi.org/10.1016/j.ecoser.2014.11.006

- García-Llorente, M.; Martín-López, B, B.; Iniesta-Arandia, I.; López-Santiago, C.; Aguilera, P. \& Montes, C. (2012). The role of multi-functionality in social preferences toward semi-arid rural landscapes: An ecosystem service approach. Environmental Science and Policy. Environmental Science \& Policy, 19-20, 136-146. https://doi.org/10.1016/j.envsci.2012.01.006.

- Hein, L.; van Koppen, K.; de Groot, R.S. \& van Ierland, E.C. (2006). Spatial scales, stakeholders and the valuation of ecosystem services. Ecological Economics, 57: 209- 228.

- Indicator Working Group. (2018). Aruba Sustainable Development Goals Baseline Measurement 2018. 113pp. Disponible en: https://census2020.aw/wpcontent/uploads/2020/09/ARUBA-SDGBASELINE-MEASUREMENT-REPORT2018.pdf

- Milcu, A.; Hanspach, J.; Abson, D. \& Fischer, J. (2013). Ecology and Society 18(3):44. http://dx.doi.org/10.5751/ES-05790-180344

- Millennium Ecosystem Assessment. (2005) Ecosystems and Human Well-being: Synthesis. Island Press, Washington, DC. ISBN 1-59726040-1. 155pp. Disponible en: https://www.millenniumassessment.org/docume nts/document.356.aspx.pdf

- National Park Aruba. (2021). Parce National Arikok Aruba. Disponible en: http://www.arubanationalpark.org/main/parkinformation/

- Hoetink, H. (2021). Aruba. Encyclopedia Britannica. Disponible en: https://www.britannica.com/place/Aruba

- Pastur, M.; Peri, P.; Lencinas, M.; GarcíaLlorente, M. \& Martín-López, B. (2016). Spatial patterns of cultural ecosystem services provision in southern patagonia. Landscape Ecology, 31(2), 383-399. https://doi.org/10.1007/s10980015-0254-9.

- Peacock, J. \& Peacock, P. (2011). Oxford Handbook of Medical Statistics. In J. Peacock, \& P. Peacock, Oxford Handbook of Medical Statistics (pp. 56-71). New York: Oxford University Press Inc.

- Plantin, J. (2014). Participatory Mapping: New Data, New Cartography. Editorial Wiley, Michigan, Estados Unidos. $160 \mathrm{p}$

- Plieninger, T.; Dijks, S.; Oteros-Rozas, E. \& Bieling, C. (2013). Assessing, mapping, and quantifying cultural ecosystem services at community level, Land use policy. Land use policy, 33, 118-129. https://doi.org/10.1016/j.landusepol.2012.12.01 3

- Ribeiro, F. P. \& Katia, T. R. (2016). Participative Mapping of Cultural Ecosystem Services in Pedra Branca State Park, Brazil. Natureza and Conservacao, 14(2), 120-127. Disponible en: https://doi.org/10.1016/j.ncon.2016.09.004

- Rumsey, D. (2016). Statistics for dummies (Segunda ed.). New York, Estados Unidos: John Wiley \& Sons Inc.

- Scholte, S.; Van Teeffelen, A. \& Verburg, P. (2015). Integrating socio-cultural perspectives into ecosystem service valuation: a review of concepts and methods. Ecological economics, 114:

67-78. https://doi.org/10.1016/j.ecolecon.2015.03.007

- Stas, K. (2016). Post-stratification or nonresponse adjustment? Survey Practice, 9(3). https://doi.org/10.29115/SP-2016-0014 
- TEEB. [The Economics of Ecosystems \& Biodiversity]. (2010a). The Economics of Ecosystems and Biodiversity: The Ecological and Economic Foundations. Chapter 1. Integrating the ecological and economic dimensions in biodiversity and ecosystem service valuation. 40 pp. Disponible en: http://doc.teebweb.org/wpcontent/uploads/Study\%20and\%20Reports/Rep orts/Ecological\%20and\%20Economic\%20Foun dations/TEEB $\% 20$ Ecological $\% 20$ and $\% 20$ Econ omic\%20Foundations\%20report/TEEB\%20Fou ndations.pdf

- TEEB. [The Economics of Ecosystems \& Biodiversity]. (2010b). Mainstreaming the Economics of Nature: A synthesis of the approach, conclusions and recommendations of TEEB. 39pp. Disponible en: http://doc.teebweb.org/wpcontent/uploads/Study\%20and\%20Reports/Re
ports/Synthesis\%20report/TEEB\%20Synthesis \%20Report\%202010.pdf

- Termansen, M., McClean, C., \& Skov-Petersen, H. (2004). Recreational site choice modelling using high-resolution spatial data. Environment and Planning, 36(6): 1085-1099.

- Turnern, R. (2015). Travel and Tourism Economic Impact 2015 Aruba. Disponible en http://www,caribbeanhotelandtourism,com/wpcontent/uploads/data_center/destinations/Aruba -WTTC-EconomicImpact2015,pdf

- Van Zanten, B. T., Verburg, P., Koetse, M., \& Van Beukering, P. (2014). Preferences for European agrarian landscapes: A meta-analysis of case studies. Land Urban Plan, 132: 89-101.

- World Bank. (2017). Population, female (\% of total population). Disponible en: http://data.worldbank.org/indicator/SP.POP.TO TL.FE.ZS $\% 20$ 